\title{
Polimorfismo rs3091244 del gen de la proteína C reactiva influencia los niveles séricos de CRP-hs en individuos de la región de La Araucanía \\ Nicolás Saavedra ${ }^{1,2}$, Angela Fallard ${ }^{1}$, Marco Araya', Pamela Serón ${ }^{1}$, Fernando Lanas ${ }^{1,2,3}$, Luis A. Salazar ${ }^{1,2}$
}

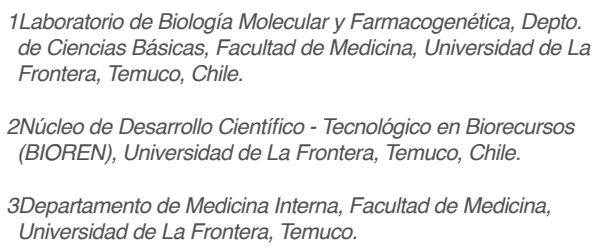

\section{Resumen:}

Introducción: Múltiples genes y sus polimorfismos han sido asociados al origen de la formación de la placa aterosclerótica y subsecuentemente el desarrollo de enfermedad cardiovascular. Uno de estos genes implicados, es el que codifica para la proteína $\mathrm{C}$ reactiva $(\mathrm{CRP})$, importante marcador proinflamatorio y de inflamación. Entre las variantes identificadas en este gen, el polimorfismo rs3040244 $\mathrm{C}>\mathrm{T}>\mathrm{A}$ ha sido asociado a elevados niveles de CRP-hs. Sin embargo, los resultados obtenidos entre poblaciones son contradictorios. Objetivo: investigar la asociación entre el polimorfismo rs3091244 y niveles séricos de CRP-hs en individuos de la región de La Araucanía.
Métodos: Se determinó la concentración sérica de CRP-hs a 157 sujetos adultos sin parentesco entre ellos.La genotipificación del polimorfismo rs3091244 se realizó mediante la técnica de PCR-RFLP.

Resultados: La distribución de genotipos para el polimorfismo rs3091244 del gen CRP fue la siguiente: CC $11.5 \%$, CT $45.2 \%$, TT $31.2 \%$, CA $5.0 \%$ y TA $7.0 \%$. Los portadores de los genotipos TT y TA presentaron elevadas concentraciones séricas de CRP-hs cuando comparadas al genotipo de referencia $C C(p=0.030$ y $p=0.002$, respectivamente).

Conclusión: Nuestros datos demuestran que el polimorfismo rs3091244 del gen CRP contribuye para el aumento de los niveles séricos de CRP-hs, y por tanto incrementa el riesgo cardiovascular. 


\section{Single nucleotide polymorphism rs3091244 of CRP gene is associated with higher levels of hs-CRP in Chilean subjects}

Background: Several genes and their polymorphisms have been associated with atherosclerotic plaque formation and subsequent development of cardiovascular disease. One of them is the C-reactive protein (CRP) encoding gene, $\mathrm{CRP}$ being a recognized important proinflammatory and inflammatory marker. Biallelics and triallelics single nucleotide polymorphisms (SNPs) in the promoter region of this gene have been identified. The rs3040244 C>T $>\mathrm{A}$ is known as the variant most strongly associated with high hs-CRP levels. However, the results between populations are contradictory. Thus, the purpose of this study was to investigate the possible association between the rs3091244 polymorphism and serum levels of hs-CRP in Southern Chilean individuals.

\section{Introducción}

Actualmente, se reconoce a la Proteína $\mathrm{C}$ reactiva (CRP), como un predictor de riesgo independiente asociado a la incidencia de enfermedades cardiovasculares $(\mathrm{ECV})^{1}$. Es así, como diversos estudios se han enfocado en clarificar el efecto directo de CRP sobre el proceso aterogénico, encontrándose acción vía disminución en la producción de óxido nítrico $(\mathrm{NO})^{2}$, estimulación de la captación de lipoproteínas de baja densidad (LDL) ${ }^{3}$, facilitación de adhesión y migración de leucocitos mediante estimulación de moléculas de adhesión ${ }^{4,5}$ e incluso ejerciendo efecto en la desestabilización de la placa aterosclerótica, mediante incremento en la expresión de la metaloproteinasa-2 (MMP-2)

Es por ello, que actualmente, la medición de los niveles de CRP-hs ha cobrado gran importancia, ya que de acuerdo a su concentración se puede categorizar el riesgo cardiovascular relativo de un individuo en bajo (CRP $<1.0$ $\mathrm{mg} / \mathrm{L})$, moderado $(1.0-3.0 \mathrm{mg} / \mathrm{L})$ y alto $(>3.0 \mathrm{mg} / \mathrm{L})^{7}$.

Adicionalmente al mejoramiento de la sensibilidad de los métodos para medir los niveles de CRP-hs sérica, el estudio de variantes en el gen que codifica CRP (CRP), emerge como una alternativa para la predicción de niveles elevados de esta proteína. CRP es codificada por un gen ubicado en el brazo largo del cromosoma 1, compuesto únicamente por 2 exones y 1 intrón, sin embargo, se trata de una región altamente polimórfica, con más de 40 variantes conocidas ${ }^{8}$. Estas, se han asociado tanto a niveles elevados como disminuidos de CRP ${ }^{9-11}$, ejemplo de ello, una repetición de dos nucleótidos (GT) con 13 alelos, dos
Methods: We determined the serum hs-CRP in 157 unrelated adult subjects. The rs3091244 polymorphism was detected by PCR-RFLP.

Results: The genotype distribution for the rs3091244 polymorphism of the CRP gene was as follows: CC $11.5 \%$, CT $45.2 \%$, TT $31.2 \%$, CA $5.0 \%$ and TA $7.0 \%$. Individuals carrying the TT and TA genotypes showed higher serum hs-CRP when compared to the reference CC genotype ( $p=0.030$ and $p=0.002$, respectively).

Conclusion: Our data shows that the rs3091244 SNP of the CRP gene contributes to increase the hsCRP levels, which may imply an increased cardiovascular risk.

Key words: hs-CRP; CRP gene; Risk factors.

de los cuales están asociados a menores valores de CRP'2 . Dentro de las variantes fuertemente asociadas a altos niveles de CRP, se encuentran dos polimorfismos de la región promotora rs3093062 y rs3091244, ambos localizados en regiones responsables de la unión de factores de transcripción. En el caso del polimorfismo rs3091244 $\mathrm{C}>\mathrm{T}>\mathrm{A}$, en presencia del alelo $\mathrm{T}$, la actividad del promotor se ve exacerbada ${ }^{13}$. Aunque se ha comprobado este efecto mediante experimentación in vitro, existe discordancia acerca de los genotipos asociados a elevados niveles de CRP-hs en distintas poblaciones.

Así, el objetivo del presente estudio fue evaluar la posible asociación entre los valores de CRP-hs y los genotipos del polimorfismo rs3091244 C > T > A en una muestra de individuos de la población general de la región de La Araucanía.

\section{Métodos}

\section{Sujetos}

Un total de 157 individuos adultos, sin ningún grado de parentesco entre sí, con edades comprendidas entre 35 y 70 años, todos provenientes de la región de La Araucanía, fueron incluidos en este estudio. Se les aplicó una encuesta para obtener información de historia familiar de enfermedad cardiovascular y de factores de riesgo tradicionales. Además, se les determinó peso, talla y se obtuvieron valores de presión arterial sistólica y diastólica. Todos los individuos, aprobaron participar en este estudio mediante la firma de un consentimiento informado, previamente aprobado por el Comité de Ética Científica del Servicio de 
Salud Araucanía Sur (Temuco, Chile).

\section{Determinaciones bioquímicas}

Se obtuvieron muestras de sangre, luego de ayuno de 12 horas, para la determinación de los niveles séricos de glucosa, colesterol total, triglicéridos y HDL-C mediante métodos enzimático-colorimétricos. La concentración de colesterol de las LDL (LDL-C) fue calculada mediante la fórmula de Friedewald. La concentración de CRP-hs fue determinada mediante método turbidimétrico (CRPhs Electa-Lab) en un analizador fotométrico Humalyzer 3000 .

\section{Genotipificación de la variante rs3091244 del gen CRP}

El ADN genómico fue extraído a partir de sangre total anticoagulada con EDTA mediante la técnica descrita por Salazar et al. ${ }^{14}$. Para la genotipificación, se amplificó un fragmento de 287 pares de bases (bp) del gen CRP mediante reacción en cadena de la polimerasa (PCR) con partidores previamente descritos ${ }^{12}$. Posteriormente, este fragmento fue sometido a restricción enzimática con las endonucleasas Hin4I y SpeI en reacciones individuales, para lograr discriminar los alelos $\mathrm{C}$ y A respectivamente, mientras que el alelo $\mathrm{T}$, se dedujo por descarte. Los productos de PCR y de restricción enzimática fueron evaluados mediante electroforesis en gel de agarosa al $2 \%$ teñido con bromuro de etidio $(0.5 \mathrm{mg} / \mathrm{L})$ y visualizado en un sistema de fotodocumentación digital.

\section{Análisis estadístico}

El análisis de los datos obtenidos se realizó utilizando el programa SigmaStat, versión 3.5 (San Rafael, EE.UU.). Las variables continuas se expresan como media \pm desviación estándar. La asociación entre variables fue analizada mediante t de Student. Además, se utilizó Chi-cuadrado ( $\chi 2)$ para el análisis de las variables no continuas y para verificar el equilibrio Hardy - Weinberg. El nivel de significancia estadística considerado en este estudio fue $\mathrm{p}<$ 0,05 .

\section{Resultados}

\section{Características de los sujetos en estudio}

Las características biodemográficas de los 157 individuos

\begin{tabular}{|c|c|}
\hline Variables & $n=157$ \\
\hline Edad, a os & $50.8 \pm 9.5$ \\
\hline Hombres, \% & 66 \\
\hline Diabetes, \% & 6 \\
\hline PAS, mm Hg & $128 \pm 22$ \\
\hline PAD, mm Hg & $79 \pm 11$ \\
\hline IMC, kg/m2 & $29.8 \pm 4.6$ \\
\hline ndice cintura / cadera & $0.92 \pm 0.08$ \\
\hline Fumadores, \% & 26 \\
\hline Hipertensi $\mathrm{n}, \%$ & 23 \\
\hline Angina, $\%$ & 1.2 \\
\hline IAM, \% & 1.2 \\
\hline CRP-hs, mg/L & $2.1 \pm 2.8$ \\
\hline Colesterol total, $\mathrm{mg} / \mathrm{dL}$ & $192 \pm 30$ \\
\hline LDL - C, mg/dL & $126 \pm 29$ \\
\hline $\mathrm{HDL}-\mathrm{C}, \mathrm{mg} / \mathrm{dL}$ & $40 \pm 9$ \\
\hline Triglic ridos, $\mathrm{mg} / \mathrm{dL}$ & $126 \pm 57$ \\
\hline Glucosa, mg/dL & $109 \pm 56$ \\
\hline
\end{tabular}

CRP-hs: Proteína C reactiva ultrasensible, IAM: infarto agudo al miocardio, IMC: índice de masa corporal, EAC: enfermedad arterial coronaria, PAS: presión arterial sistólica, PAD: presión arterial diastólica, HDL-C: Colesterol de las HDL, LDL-C: Colesterol de las $L D L$.

\begin{tabular}{|c|c|c|c|c|}
\hline Riesgo Relativo & $\begin{array}{l}\text { Bajo } \\
<1.0 \\
(n=74)\end{array}$ & $\begin{array}{c}\text { Moderado } \\
1.0-3.0 \\
(n=58)\end{array}$ & $\begin{array}{c}\text { Alto } \\
>3.0 \\
(n=25)\end{array}$ & $\mathbf{P}^{*}$ \\
\hline Mujeres, \% & 37 & 31 & 24 & 0.462 \\
\hline Hombres, \% & 63 & 69 & 76 & \\
\hline Diabetes mellitus, \% & 2 & 8 & 12 & 0.213 \\
\hline PAS, $\mathrm{mm} \mathrm{Hg}$ & $125 \pm 25$ & $129 \pm 19$ & $133 \pm 23$ & 0.320 \\
\hline $\mathrm{PAD}, \mathrm{mm} \mathrm{Hg}$ & $77 \pm 12$ & $80 \pm 11$ & $81 \pm 11$ & 0.223 \\
\hline IMC, $\mathrm{Kg} . / \mathrm{m} 2$ & $28.5 \pm 3.2$ & $30.3 \pm 4.9$ & $32.9 \pm 6.3$ & $<0.001$ \\
\hline ndice Cintura/Cadera & $0.91 \pm 0.09$ & $90.92 \pm 0.07$ & $0.94 \pm 0.10$ & 0.380 \\
\hline Glucosa, mg/dL & $96 \pm 19$ & $109 \pm 70$ & $158 \pm 101$ & 0.406 \\
\hline Colesterol total, $\mathrm{mg} / \mathrm{dL}$ & $181 \pm 27$ & $208 \pm 25$ & $168 \pm 38$ & 0.077 \\
\hline LDL-C, mg/dL & $115 \pm 27$ & $142 \pm 21$ & $112 \pm 46$ & 0.131 \\
\hline HDL-C, mg/dL & $43 \pm 8$ & $39 \pm 11$ & $35 \pm 3$ & 0.468 \\
\hline Triglic ridos, $\mathrm{mg} / \mathrm{dL}$ & $115 \pm 59$ & $142 \pm 60$ & $109 \pm 47$ & 0.609 \\
\hline
\end{tabular}

CRP-hs, Proteína $C$ reactiva ultrasensible; IMC, índice de masa corporal; EAC, enfermedad arterial coronaria; PAS, presión arterial sistólica; PAD, presión arterial diastólica; $H D L-C$, Colesterol de las HDL; LDL-C, Colesterol de las LDL. Valores de CRP-hs en mg/L. *Test de ANOVA o Chi-cuadrado. 


\begin{tabular}{|c|c|c|c|c|c|c|c|c|}
\hline & \multicolumn{4}{|c|}{ Genotipos } & \multicolumn{4}{|c|}{ Alelos } \\
\hline CC & CT & TT & CA & TA & AA & C & $\mathbf{T}$ & A \\
\hline $11.5 \%$ & $45.2 \%$ & $31.2 \%$ & $5 \%$ & $7 \%$ & $0 \%$ & 0.370 & 0.570 & 0.060 \\
\hline (18) & (71) & (49) & (8) & (11) & (0) & & & \\
\hline
\end{tabular}

Equilibrio de Hardy-Weinberg: $C>T>A ; \chi 2=1.02(p=N S)$.

Número de individuos en paréntesis.

\begin{tabular}{|c|c|c|c|c|}
\hline \multicolumn{5}{|c|}{ Genotipos } \\
\hline CC & CT & TT & CA & TA \\
\hline (18) & (71) & (49) & (8) & (11) \\
\hline $1.17 \pm 0.79^{a, b, c}$ & $2.10 \pm 2.89$ & $2.90 \pm 3.07$ & $2.44 \pm 3.79$ & $3.56 \pm 2.63$ \\
\hline
\end{tabular}

Valores en $\mathrm{mg} / \mathrm{L}$, presentados como media $\pm D E$. Número de individuos en paréntesis.

a $p=0.030$ vs. Genotipo TT

$b p=0.002$ vs. Genotipo TA

c $p=0.022$ vs. Genotipos CA/TA

investigados en este estudio, se resumen en la Tabla 1. En la Tabla 2, se puede apreciar la categorización de riesgo cardiovascular de los sujetos estudiados, según niveles de CRP-hs; encontrándose diferencias significativas entre los grupos de alto, moderado y bajo riesgo, sólo para los valores de IMC.

\section{Genotipificación de la variante rs3091244 del gen CRP}

La distribución genotípica y la frecuencia relativa de los alelos para el polimorfismo estudiado del gen CRP son mostradas en la Tabla 3. La distribución de genotipos fue la esperada según el equilibrio de Hardy-Weinberg. Además, se analizó la concentración de CRP-hs para cada genotipo, observándose diferencias significativas al comparar los niveles entre los portadores del genotipo de referencia $(\mathrm{CC})$ y los genotipos $\mathrm{TT}(\mathrm{p}=0.030), \mathrm{TA}(\mathrm{p}=0.002)$, $\mathrm{CA} / \mathrm{TA}(\mathrm{p}=0.022)$, presentando estos últimos, niveles elevados de CRP-hs (Tabla 4).

\section{Discusión}

CRP es un importante marcador inflamatorio que ejerce una acción directa en el proceso de aterogénesis y es considerado marcador de riesgo de ECV, pudiendo ser estratificado según sus niveles plasmáticos. Al hacer la clasificación del riesgo cardiovascular de los individuos estudiados, según los diferentes niveles de CRP-hs y relacionarlos con las características clínicas y de laboratorio, se asoció los niveles de CRP-hs por sobre los $3 \mathrm{mg} / \mathrm{L}$ a un mayor valor de IMC, datos semejantes a los encontrados por otro grupo con anterioridad ${ }^{15} \mathrm{y}$ que pudieran ser consecuencia de un fenómeno inflamatorio asociado a obesidad, siendo IL-6, secretada por adipocitos, sindicada como activador de la síntesis de CRP en el hígado $^{16,17}$. En otros de los parámetros estudiados, se observa una tendencia al alza (PAS, PAD, glucosa) o a la baja (HDL), sin embargo, no mostraron diferencias estadísticamente significativas entre las tres categorías de riesgo.

En el presente estudio, se evaluó por primera vez en el país, la asociación entre los niveles séricos de CRP-hs y el polimorfismo rs3091244, una de las variantes fuertemente relacionada al aumento de $\mathrm{CRP}^{18}$, sin embargo, el genotipo determinante de esta asociación ha mostrado ser distinto entre poblaciones ${ }^{8,19,20}$.

Nuestros hallazgos evidencian una predominancia en la frecuencia relativa del alelo $\mathrm{T}$ (0.570), y del genotipo CT (45.2\%) por sobre los demás genotipos encontrados (TT: $31.2 \%$, CC: $11.5 \%$, TA: $7 \%$ y CA: $5 \%$ ), además de la au- 
sencia del genotipo AA. Se observan discrepancias entre la distribución genotípica y alélica con estudios anteriormente publicados $^{13,18}$, debido, probablemente, a las diferencias étnicas de las poblaciones analizadas.

$\mathrm{Al}$ analizar los valores de CRP-hs entre los portadores del genotipo $\mathrm{CC}$ versus $\mathrm{CA} / \mathrm{TA}$ se observó una diferencia significativa ( $\mathrm{p}=0.022)$, comprobándose mayores niveles de CRP-hs en individuos que presentan los alelos A y/o T comparado con el alelo C (alelo normal o de referencia); resultado similar al obtenido por otros autores que asocian los mismos alelos a niveles elevados de CRP-hs ${ }^{21,22}$. El mecanismo que explicaría los mayores niveles de CRP-hs en estos individuos, podría ser atribuido a una mayor actividad del promotor del gen CRP en presencia de dichos alelos, traduciéndose en mayores niveles plasmáticos de $\mathrm{CRP}^{23}$ y consecuentemente mayor riesgo cardiovascular. Entre las limitaciones que presenta nuestro estudio, pode- mos comentar que sólo se realizó una medición de CRPhs a cada individuo, siendo conveniente para calcular las cifras de CRP-hs, realizar dos determinaciones separadas entre 1 a 3 semanas $^{7,24}$. Sin embargo, asumimos el sesgo de una única medición, considerando que la CRP-hs presenta escasa fluctuación a corto plazo ${ }^{25}$. Además, de acuerdo a lo encontrado por Ridker et al. ${ }^{26}$, una única determinación puede ser útil como predictor de enfermedad clínica.

En resumen, nuestro estudio muestra una asociación entre la variante rs3091244 del gen CRP con niveles elevados de CRP-hs, por lo que podría ser útil como marcador molecular de riesgo cardiovascular, considerando que una elevación en los niveles de CRP significa mayor riesgo para el desarrollo de patologías del aparato circulatorio. Sin embargo, ello debiera corroborarse con otros estudios a lo largo del país, para poder extrapolar estos hallazgos.

\section{Referencias:}

1. BUCKLEY DI, FU R, FREEMAN M, ROGERS K, HELFAND M. C-reactive protein as a risk factor for coronary heart disease: a systematic review and meta-analyses for the U.S. Preventive Services Task Force. Ann Intern Med. 2009; 151: 483-495.

2. CLAPP BR, HIRSCHFIELD GM, STORRY C, GALLIMORE JR, STIDWILL RP, SINGER M, et al. Inflammation and endothelial function: direct vascular effects of human C-reactive protein on nitric oxide bioavailability. Circulation. 2005; 111: 1530-1536.

3. ZWAKA TP, HOMBACH V, TORZEWSKI J. C-reactive protein-mediated low density lipoprotein uptake by macrophages: implications for atherosclerosis. Circulation. 2001; 103: 1194-1197.

4. MONTECUCCO F, STEFFENS S, BURGER F, PELLI G, MONACO C, MACH F. C-reactive protein (CRP) induces chemokine secretion via CD11b/ICAM-1 interaction in human adherent monocytes. J Leukoc Biol. 2008; 84: 11091119.

5. PAUL A, KO KW, LI L, YECHOOR V, MCCRORY MA, SZALAI AJ, et al. C-reactive protein accelerates the progression of atherosclerosis in apolipoprotein E-deficient mice. Circulation. 2004; 109: 647-655.

6. DORONZO G, RUSSO I, MATTIELLO L, TROVATI M,
ANFOSSI G. C-reactive protein increases matrix metalloproteinase-2 expression and activity in cultured human vascular smooth muscle cells. J Lab Clin Med. 2005; 146: 287-298.

7. PEARSON TA, MENSAH GA, ALEXANDER RW, ANDERSON JL, CANNON RO, CRIQUI M, et al. Markers of inflammation and cardiovascular disease: application to clinical and public health practice: A statement for healthcare professionals from the Centers for Disease Control and Prevention and the American Heart Association. Circulation. 2003; 107: 499-511.

8. HAGE FG, SZALAI AJ. C-reactive protein gene polymorphisms, C-reactive protein blood levels, and cardiovascular disease risk. J Am Coll Cardiol. 2007; 50:1115-1122,

9. ZEE RY, RIDKER PM. Polymorphism in the human Creactive protein (CRP) gene, plasma concentrations of $\mathrm{CRP}$, and the risk of future arterial thrombosis. Atherosclerosis. 2002; 162: 217-219.

10. BRULL DJ, SERRANO N, ZITO F, JONES L, MONTGOMERY HE, RUMLEY A, et al. Human CRP gene polymorphism influences CRP levels: implications for the prediction and pathogenesis of coronary heart disease. Arterioscler Thromb Vasc Biol. 2003; 23: 2063-2069.

11. KOVACS A, GREEN F, HANSSON LO, LUNDMAN P, SAMNEGARD A, BOQUIST $S$, et al. A novel common single nucleotide polymorphism in the promoter region of the $\mathrm{C}$-reactive protein gene associated with the plasma 
concentration of C-reactive protein. Atherosclerosis. 2005; 178:193-198

12. RUSSELL AI, CUNNINGHAME GRAHAM DS, SHEPHERD C, ROBERTON CA, WHITTAKER J, MEEKS $\mathrm{J}$, et al. Polymorphism at the C-reactive protein locus influences gene expression and predisposes to systemic lupus erythematosus. Hum Mol Genet. 2004; 13:137-147.

13. SZALAI AJ, WU J, LANGE EM, MCCRORY MA, LANGEFELD CD, WILLIAMS A, et al. Single-nucleotide polymorphisms in the C-reactive protein (CRP) gene promoter that affect transcription factor binding, alter transcriptional activity, and associate with differences in baseline serum CRP level. J Mol Med. 2005; 83:440-447.

14. SALAZAR LA, HIRATA MH, CAVALLI SA, MACHADO MO, HIRATA RD. Optimized procedure for DNA isolation from fresh and cryopreserved clotted human blood useful in clinical molecular testing. Clin Chem. 1998; 44:1748-1750.

15. SESHADRI P, IQBAL N, STERN L, WILLIAMS M, CHICANO KL, DAILY DA, et al. A randomized study comparing the effects of a low-carbohydrate diet and a conventional diet on lipoprotein subfractions and C-reactive protein levels in patients with severe obesity. Am J Med. 2004;117:398-405.

16. SHOELSON SE, LEE J, GOLDFINE AB. Inflammation and insulin resistance. J Clin Invest. 2006;116:1793-1801.

17. BLOOMGARDEN ZT. Inflammation and insulin resistance. Diabetes Care. 2003; 26:1922-1926.

18. CRAWFORD DC, SANDERS CL, QIN X, SMITH JD, SHEPHARD C, WONG M, et al. Genetic variation is associated with C-reactive protein levels in the Third National Health and Nutrition Examination Survey. Circulation. 2006; 114:2458-2465.

19. KIVIMAKI M, LAWLOR DA, SMITH GD, EKLUND C, HURME M, LEHTIMAKI T, et al. Variants in the CRP gene as a measure of lifelong differences in average C- reactive protein levels: the Cardiovascular Risk in Young Finns Study, 1980-2001. Am J Epidemiol. 2007; 166:760764.

20. EKLUND C, KIVIMAKI M, ISLAM MS, JUONALA M, KAHONEN M, MARNIEMI J, ET al. C-reactive protein genetics is associated with carotid artery compliance in men in The Cardiovascular Risk in Young Finns Study. Atherosclerosis. 2008; 196:841-848.

21. WANG Q, HUNT SC, XU Q, CHEN YE, PROVINCE MA, ECKFELDT JH, et al. Association study of CRP gene polymorphisms with serum CRP level and cardiovascular risk in the NHLBI Family Heart Study. Am J Physiol Heart Circ Physiol. 2006; 291:H2752-2757

22. KOMURCU-BAYRAK E, ERGINEL-UNALTUNA N, ONAT A, OZSAIT B, EKLUND C, HURME M, et al. Association of C-reactive protein (CRP) gene allelic variants with serum CRP levels and hypertension in Turkish adults. Atherosclerosis. 2009; 206:474-479.

23. KATHIRESAN S, LARSON MG, VASAN RS, GUO CY, GONA P, KEANEY JF, et al. Contribution of clinical correlates and $13 \mathrm{C}$-reactive protein gene polymorphisms to interindividual variability in serum C-reactive protein level. Circulation. 2006; 113:1415-1423.

24. GÓMEZ A. Inflamación, hemostasia y enfermedad coronaria. La proteína $\mathrm{C}$ reactiva y el dímero $\mathrm{D}$ como marcadores del proceso de arteriosclerosis. Clin Invest Arterioscl. 2002; 14:242-257.

25. MACY EM, HAYES TE, TRACY RP. Variability in the measurement of C-reactive protein in healthy subjects: implications for reference intervals and epidemiological applications. Clin Chem. 1997; 43:52-58.

26. RIDKER PM, HENNEKENS CH, BURING JE, RIFAI N. $\mathrm{C}$-reactive protein and other markers of inflammation in the prediction of cardiovascular disease in women. $\mathrm{N}$ Engl J Med. 2000; 342:836-843. 